\title{
PET/MRI: Methodology and Clinical Applications Clinical Applications of SPECT-CT
}

I. Carrio and P. Ros, Eds.

New York, NY: Springer, 2014, 163 pages, $\$ 179$

Following the success of PET/CT in clinical applications, it would seem logical that PET/MR imaging can provide an innovative alternative taking full advantage of the superiority of MR imaging over CT in differentiating soft-tissue abnormalities while reducing radiation exposure. PET/MR imaging combines the molecular data of PET with the precise anatomic and physiologic information of MR imaging and offers the promise of a simplified workflow and pathway for molecular imaging in medicine.

In compiling this small textbook, the editors have brought in an international group of 31 experts in the fields of PET and MR imaging. There are 11 chapters divided into 3 parts. The first part deals with methodology and equipment and comprises 3 chapters: PET/MR imaging system design; image distortions, including artifacts in clinical PET/MR imaging; and workflow and practical logistics, including imaging protocols. The second part, with 6 chapters, discusses the clinical applications and potential roles of PET/MR imaging in oncology, cardiology, and neurology. PET/MR imaging of breast cancer, lymphoma, liver disease, colorectal cancer, brain imaging, and cardiology is covered in individual chapters. Unfortunately, there are only a few illustrative clinical cases, but the images are clear and informative. The last part, with 2 chapters, addresses the risks and safety of PET/ MR imaging, health-care costs, and the impact of PET/MR imaging. The references are current, but the index seems limited. There is no preface or foreword.

This book is recommended to trainees and practitioners in the fields of nuclear medicine, radiology, and radiologic sciences who want to learn more about the latest developments and clinical utility of a new hybrid, PET/MR imaging.

\author{
E. Edmund Kim \\ University of California at Irvine \\ 101 The City Dr. S. \\ Orange, $C A 92868$ \\ E-mail: edmundek@uci.edu
}

DOI: 10.2967/jnumed.114.148676
H. Ahmadzadehfar and H.-J. Biersack, Eds.

New York, NY: Springer, 2014, 304 pages, \$229

Now that hybrid imaging is a well-established part of virtually all nuclear medicine studies, there is a great need for a clear understanding of how to perform and use this type of imaging. SPECT/CT cameras have considerably improved diagnostic accuracy in recent years, with better localization and characterization of radionuclide abnormalities as well as additional detection of anatomically small lesions. Furthermore, CT coregistration provides superior quantification of radiotracer uptake on the basis of the attenuation correction capabilities of CT.

This book, with 14 chapters, provides such a resource and covers the full spectrum of clinical applications of SPECT/CT in diagnosis and treatment planning of benign and malignant diseases. It was written by 25 contributors to be a practical guide to the clinical utility of SPECT/CT. The first and second chapters discuss the physics and technology of SPECT/CT and its use in dosimetry. The application of SPECT/CT in the imaging of a range of pathologic conditions is then addressed in the following 9 chapters. The clinical applications that are covered include imaging of the thyroid, parathyroid, bone, coronary artery, and lung. The final 3 chapters are devoted to internal radiation therapy, Bremsstrahlung SPECT/CT, and imaging of the brain and inflammation. Each chapter includes an introduction and then covers the techniques, indications, clinical applications, and limits of SPECT/CT. The illustrative images are clear and informative, and the tables are useful. The references in each chapter are current, and the index is helpful.

Readers will find this book to be an essential and useful reference on the clinical applications of SPECT/CT. I highly recommend it to trainees and practitioners in nuclear medicine, radiology, and radiologic sciences.

\author{
E. Edmund Kim \\ University of California at Irvine \\ 101 The City Dr. S. \\ Orange, CA 92868 \\ E-mail: edmundek@uci.edu
}

DOI: 10.2967/jnumed.114.148684 